Objectives: To determine the strength of the relationship between SU and the incidence of gout flares and thereby to determine whether $\mathrm{SU}$ is a valid surrogate endpoint according to the BSES3 framework.

Methods: A systematic search of Medline via PubMed, the Cochrane Central Register of Controlled Trials, EMBase via OVID, the Institute for Scientific Information Web of Science, and other sources identified relevant studies. Standardised data elements were extracted by 2 independent reviewers (LS, $\mathrm{MBM}$ ) and disagreements resolved by discussion (RC). Eligible trials were parallel-group, randomised trials of ULT of at least 3 months duration in people with gout. For the meta-regression analysis, a mixed linear model was used to combine the ratio of flare rates between groups (SAS software, v9.4 for Windows). Trials with multiple arms were treated as individual trials, referred to as "randomised comparisons" (i.e., 3 -arm trials with 2 active interventions was handled as 2 randomised comparisons). Data was analysed by meta-regression using the between-arm difference in proportion of individuals who achieved target $\mathrm{SU}(<0.36 \mathrm{mmol} / \mathrm{L})$ as independent variable from at 3 months (or 6 and 12 months if 3 month values not available) against flare rate (dependent variable).

Results: After screening 234 abstracts, 82 trials were scrutinized, of which 9 trials (with 16 comparisons) met inclusion criteria. A total of 5,696 people with gout entered into the meta-regression model. The longest RCT included was only 12 months duration. The pooled Odds Ratio (OR) suggested a small but statistically significant favourable association between the active and comparator urate lowering therapies and flare frequency (OR, $0.83 ; 95 \% \mathrm{Cl} 0.70$ to 0.99$]$ ). Substantial heterogeneity was present (between trial variance: $0.07 ; 0.03$ to 0.30 ). Meta-regression analysis did not reveal any statistically significant association between the proportion of individuals who achieved target SU and the observed flare rate $(P=0.82)$; the model fit did not improve after inclusion of the covariate into the model (between trial variance: $0.08 ; 0.03$ to 0.33 ) (Figure).

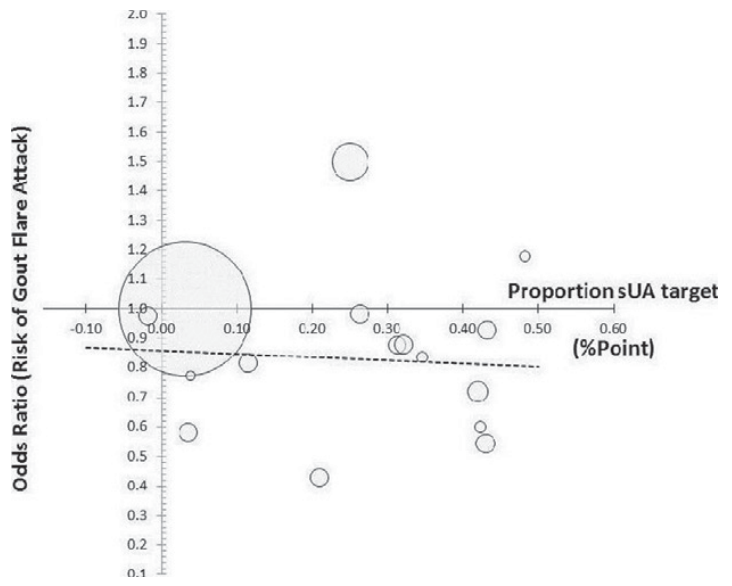

Conclusions: Substituting surrogate endpoints (proportion achieving target $\mathrm{SU}$ ) for the important clinical outcome (gout flares) allows conduct of shorter smaller trials. However, based on aggregate trial-level data (meta-regression) an anticipated association between SU and gout flare could not be confirmed. Trial duration may have been too short to observe a reduction in flares and further work using data from long-term extension studies is underway.

Disclosure of Interest: None declared

DOI: 10.1136/annrheumdis-2017-eular.1544

\section{OP0268 NURSE-LED CARE VERSUS GENERAL PRACTITIONER CARE OF PEOPLE WITH GOUT: A UK COMMUNITY-BASED RANDOMISED CONTROLLED TRIAL}

M. Doherty ${ }^{1}$, W. Jenkins ${ }^{1}$, H. Richardson ${ }^{1}$, A. Abhishek ${ }^{1}$, D. Ashton ${ }^{1}$, C. Barclay ${ }^{1}$, L. Duley ${ }^{2}$, H. Jones ${ }^{1}$, M. Santarelli ${ }^{1}$, A. Sarmanova ${ }^{1}$, M. Stevenson ${ }^{3}$, W. Zhang ${ }^{1}{ }^{1}$ Division of Rheumatology, Orthopedics and Dermatology; ${ }^{2}$ Clinical Trials Unit, University of Nottingham, Nottingham; ${ }^{3}$ Health Economics and Decision Science, University of Sheffield, Sheffield, United Kingdom

Background: Despite increasing prevalence of gout in the UK (1), a variety of barriers result in suboptimal care $(1,2)$ with only $40 \%$ of gout patients receiving urate-lowering therapy (ULT), usually at fixed dose without titration to a serum uric acid (SUA) target $(1,2)$. Nurses successfully manage many chronic diseases in the community, and we have shown that when people with gout are fully informed and involved in management decisions uptake of ULT is high and subsequent adherence under nurse-led care is excellent (3).

Objectives: To directly compare nurse-led care to general practitioner (GP) care of people with gout in a 2 year randomised controlled trial (NIHR CRN Portfolio No.12943)

Methods: 517 participants with acute gout in the previous year were identified from 56 local GP practices and randomised to nurse-led or continuing GP care. The nurses were trained about gout and its management according to recommended best practice (EULAR and BSR guidelines) involving full information, addressing illness perceptions, and involving patients in management decisions. Assessments were undertaken at 1 and 2 years. Analysis was intention to treat (last observation carried forward).

Results: Nurse $(n=255)$ and GP ( $n=262)$ groups were well matched at baseline for mean age (62 v 64yrs), sex ( $90 \% \vee 89 \%$ men), mean disease duration $(11.6$ $v 12.7 \mathrm{yrs})$, mean gout attack frequency in prior year (4.2 v. 3.8), tophi $(13.7 \%$ v. $8.8 \%)$, mean SUA ( 443 v. $439 \mu \mathrm{mol} / \mathrm{L})$, mean eGFR (71.5 v. 70.2) and ULT use $(40 \%$ v. $39 \%)$ (all $p>0.05)$. By 2yrs, $22(8.6 \%)$ and $54(20.6 \%)$ participants had discontinued the nurse and GP groups $(p<0.001)$, including 2 v. 8 deaths respectively. Comparing nurse and GP groups at 2 yrs: $95 \%$ v. $29 \%$ had SUA $<360 \mu \mathrm{mol} / \mathrm{L}$ (primary outcome); $88 \%$ v. $16 \%$ had SUA $<300 \mu \mathrm{mol} / \mathrm{L}$; mean (SD) SUA was $252 \pm 73$ v. $418 \pm 106 ; 97 \%$ v. $54 \%$ were on ULT; and mean (SD) dose of allopurinol was 470 (140) v. 240 (107) mg/day (all $p<0.001)$. Mean (SD) attack frequency during the 2nd year was $0.33(0.93)$ in the nurse v. $0.94(2.03)$ in the GP group $(p<0.001)$, and at $2 y$ rs tophi were present in $2.6 \%$ (reduced) $v$. $9.6 \%$ respectively $(p<002)$. Although equivalent at baseline, mean (SD) SF-36 norm-based physical component scores were better at 2yrs in the nurse group (41.31 (16.76) v. 37.87 (14.31); $p<0.05)$.

Conclusions: Nurse-led care of people with gout in the UK community can result in high uptake and excellent adherence to ULT over a 2yr period, achievement of target SUA in $>9 / 10$ cases and consequent improvements in patient-centred outcomes and quality of life. This study reinforces the benefits of "treat-to-target". Compared to standard GP care this model is likely to be cost effective long-term and merits further consideration.

References:

[1] Doherty M. et al. Ann Rheum Dis 2012;71:1765-70.

[2] Kuo C-F. et al. Ann Rheum Dis 2015;74:661-7.

[3] Rees F. et al. Ann Rheum Dis 2013:72:826-30.

Acknowledgements: Arthritis Research UK (Award No.19703) funded this study.

Disclosure of Interest: M. Doherty Grant/research support from: AstraZeneca, Consultant for: AstraZeneca, Grunenthal, Mallinckrodt and Roche, W. Jenkins: None declared, H. Richardson: None declared, A. Abhishek Grant/research support from: AstraZeneca, D. Ashton: None declared, C. Barclay: None declared, L. Duley: None declared, H. Jones: None declared, M. Santarelli: None declared, A. Sarmanova: None declared, M. Stevenson: None declared, W. Zhang Consultant for: AstraZeneca and Grunenthal DOI: 10.1136/annrheumdis-2017-eular.5006

\section{OP0269 EFFECT OF XANTHINE OXIDASE INHIBITORS ON THE INCIDENCE OF CARDIOVASCULAR EVENTS: A SYSTEMATIC REVIEW AND META-ANALYSIS OF RANDOMISED CONTROLLED TRIALS}

M. Bredemeier, L.M. Lopes, M.A. Eisenreich, G.K. Bongiorno, S. Hickmann. Rheumatology Division, Hospital Nossa Senhora da Conceição - Grupo Hospitalar Conceição, Porto Alegre - RS, Brazil

Background: There is evidence that xanthine oxidase inhibitors (XOI) may reduce the risk of major adverse cardiovascular events (MACE) $(1,2)$ and lower blood pressure (3). To date, this evidence is based mainly on observational studies (2). Objectives: To compare the incidence of MACE, mortality, and total and specific cardiovascular $(\mathrm{CV})$ events in patients enrolled in randomized controlled trials (RCTs) comparing XOI with placebo or no treatment.

Methods: A systematic review (CRD42015016073) searching for RCTs using PubMed, EMBASE, Cochrane Library, Web of Science, and Lilacs databases, and hand searching, was ended in Dec 2016. All RCTs comparing XOls with placebo or no treatment lasting $\geq 4$ weeks and including only adult individuals were eligible. The primary outcomes were the incidence of MACE (CV death, non-fatal myocardial infarction, unstable angina requiring urgent revascularization, or non-fatal stroke) and mortality; total CV events (TCE), specific CV outcomes, and serious adverse events (SAE) served as secondary outcomes. Associations were tested using the Peto odds ratio $(\mathrm{OR})$ without zero-cell continuity correction.

Results: In total, 81 studies including approx, 11,000 individuals reported extractable data on $\mathrm{CV}$ events. The use of $\mathrm{XOI}$ tended to be associated with lower incidence of MACE (OR=0.64, 95\% Cl 0.41-1.01, $\mathrm{P}=0.056)$, but not with mortality $(0.95,0.63-1.44)$. However, there was a significantly reduced incidence of TCE $(0.66,0.54-0.80, P<0.001)$, especially new/worsening hypertension $(0.57$, $0.37-0.87, P=0.009$ ), and a trend for reduction in the incidence of new/worsening heart failure $(0.74,0.53-1.04, P=0.086)$. The incidence of SAE $(0.86,0.71-1.05)$ did not differ significantly. Subgroup analysis suggested a protective effect for MACE in studies with high prevalence $(>50 \%)$ of cardiac diseases $(0.52,0.30-$ $0.91, P=0.021$ ). Sensitivity analysis excluding studies at high or unknown risk of bias produced no significant change in results, but reinforced the association with reduced incidence of hypertension $(0.26,0.11-0.60, \mathrm{P}=0.001)$ and heart failure $(0.55,0.32-0.94, P=0.030)$. Separate analysis of data on purine-like $\mathrm{XOI}$ (allopurinol and oxypurinol) confirmed the results of the primary analysis. Exploratory metaregression analysis showed association of dose of allopurinol with higher incidence of TCE $(P=0.023$, random effects $)$ and SAE $(P<0.001$, see Figure 1). Accordingly, in the subgroup with doses $\leq 300 \mathrm{mg} /$ day of allopurinol, a reduction of incidence of MACE $(0.36,0.18-0.68, P=0.002)$, TCE $(0.38,0.26-$ $0.54, \mathrm{P}<0.001)$, and $\mathrm{SAE}(0.49,0.34-0.71, \mathrm{P}<0.001)$ was observed, while the $\mathrm{SAE}$ risk increased in doses $>300 \mathrm{mg} /$ day $(1.39,1.04-1.91, P=0.047)$. There was 